\title{
A Transcriptional Regulatory System of the S. cerevisiae OLE1 Gene Responds to Fatty Acid Species and Intracellular Amount, and not Simply Membrane Status
}

\author{
Marshall Willey (D, Matt Ochs, Clara Busse, and Virginia McDonough \\ Department of Biology, Hope College, PO Box 9000, Holland, MI 49422-9000, USA \\ Correspondence should be addressed to Virginia McDonough; mcdonough@hope.edu
}

Received 7 June 2019; Revised 3 August 2019; Accepted 12 August 2019; Published 25 January 2020

Academic Editor: Clifford A. Lingwood

Copyright ( 2020 Marshall Willey et al. This is an open access article distributed under the Creative Commons Attribution License, which permits unrestricted use, distribution, and reproduction in any medium, provided the original work is properly cited.

\begin{abstract}
We examined the effects of unsaturated fatty acid (UFA) species and their concentration on the expression of OLE1, which encodes the stearoyl CoA desaturase, in Saccharomyces cerevisiae. We controlled the amount of UFA taken up by the cell by varying the concentration of tergitol in the medium. When cultured with $1 \mathrm{mM}$ fatty acid in $0.1 \%$ tergitol, cells took up much more fatty acid than when cultured with the same concentration of fatty acid at $1 \%$ tergitol, although the amount incorporated was dependent on UFA species. For each fatty acid tested, we found that the higher uptake ( $0.1 \%$ tergitol condition) had a stronger impact on OLE1 regulation. A principal product of the desaturase 16:1 $\Delta 9$, and the nonnative UFA 18:2 $\Delta 9,12$, most strongly repressed the reporter construct OLE1-lacZ transcription, while the other major product of the desaturase, 18:1 $\Delta 9$, and the nonnative UFA 17:1 10 caused a more diminished response. Based on these results, our initial hypothesis was that OLE1 was regulated in response to membrane fluidity; however, subsequent work does not support that idea; we have found that conditions that affect membrane fluidity such as growth temperature and growth with saturated or trans fatty acid supplementation, do not regulate OLE1 in the direction predicted by fluidity changes. We conclude that at least one signal that regulates OLE1 transcriptional expression is most likely based on the fatty acids themselves.
\end{abstract}

\section{Introduction}

In the yeast Saccharomyces cerevisiae, there is a single fatty acid desaturase encoded by the OLE1 gene [1,2]. The stearoyl-CoA desaturase, which functions as a homodimer [3], introduces a cis double bond between carbons 9 and 10 of the acyl chain. Its primary products in vivo are palmitoleic $(16: 1 \Delta 9)(\mathrm{x}: \mathrm{y} \Delta \mathrm{n}$, fatty acids containing $\mathrm{x}$ carbon atoms and $\mathrm{y}$ cis double bonds located at position $\mathrm{n}$ from the carboxyl end; UFA, unsaturated fatty acid) and oleic acid (18:1 $\Delta 9)$ [4]. There is accumulating evidence that aberrant expression of the stearoyl-CoA desaturase is also involved in disease; in mammals, an anticancer gene ORCTL3 was identified that could cause apoptosis in certain transformed cells [5]. Later research identified the target of ORTLC3 as the stearoyl-CoA desaturase [6]. And in yeast, overexpression of stearoyl-CoA desaturase leads to invasive growth [7]. Recent investigations have identified a role of $A R V 1$ in fatty liver disease [8]. The yeast arv1 mutants are defective in OLE1 regulation, implying a possible connection between fatty liver disease and desaturase expression. Recently, Vincent et al. have demonstrated the potential of the desaturase as a druggable target for treatment of neurodegenerative disorder such as Parkinson's disease [9].

OLE1 expression is regulated at the level of transcription, RNA stability and protein activity (reviewed in $[10,11]$ and is known to be regulated by various growth conditions, including exogenous unsaturated fatty acids [12-14], cobalt and carbon source [15], ethanol concentration [16, 17], and oxygen [18]. While environmental conditions such as culture temperature have been investigated, these studies have generally found transient changes in expression with a small long term impact on fatty acid composition $[19,20]$. The best characterization of these regulatory systems is the positive regulation of OLE1 when cells are grown in the absence of products of the desaturase: UFAs containing a double bond between carbons 9 and 10 . Previous work has determined that transcription of the OLE1 gene is controlled, at least in part, by homologous transcription factors, encoded by the genes MGA2 and SPT23 [21]. When 
there are abundant UFAs, the Mga2p and Spt23p transcription factors are tethered as inactive p120 forms in the endoplasmic reticulum membrane. But, when UFAs are limiting, these two proteins are proteolytically cleaved from their carboxy tails, and the soluble p90 N-termini are translocated into the nucleus, where they can activate transcription of the OLE1 gene [21].

The processing of Mga2p and Spt23p into their active p90 forms occurs through a controlled, ubiquitin-regulated proteolysis by the proteasome in the endoplasmic reticulum. A complex of the proteins Cdc48p-Npl4p-Ufd1p is required for this activation of Mga2p and Spt23p [22-24]. In addition, the UBX2 encoded protein has been shown to be important in the regulation of $O L E 1$ in response to exogenous fatty acids, acting as a bridging factor between the cytosolic Cdc48p-Npl1p-Ufd1p complex with the membrane associated ubiquitin ligase complex and Mga2p and Spt23p $[25,26]$. In addition to its role in regulating OLE1, Mga2p has been implicated in regulation of at least one other gene, ERG1, which is involved in sterol biosynthesis [27]. Interesting work by Covino et al. has shown that the when Mga2p forms a homodimer, the orientation of the transmembrane helices can vary based on the lipid environment. The alternative conformations are stabilized differentially, depending on whether the protein is found in a membrane rich or poor in 18:2 $\Delta 9,12$. These alternative conformations result in differing abilities of Mga2p to be processed into the active $\mathrm{p} 90$ form, hence allowing Mga2p to act as a lipid sensor [28].

However, UFAs are diverse in structure; varying by chain length, presence or absence of double bonds in the acyl chain, number and location of double bonds and whether the bonds are cis or trans configuration. We investigated whether some of these distinctions resulted in different regulatory schemes to control OLE1 expression. In this work, we look at the effect of concentration and UFA structure of four UFAs on controlling desaturase expression: the authentic products of the desaturase, palmitoleic acid (16:1 $\Delta 9)$ and oleic acid (18:1 $\Delta 9)$, and two nonnative products linoleic acid $(18: 2 \Delta 9,12)$, a polyunsaturated fatty acid with two double bonds, between carbons $9-10$ and 12-13, and the fatty acid cis-10-heptadecenoic acid $(17: 1 \Delta 10)$, an odd chain length monounsaturated fatty acid which does not contain a $9-10$ bond, but a $10-11$ bond. These fatty acids were carefully chosen so that we could examine how the various structural and physical characteristics affect regulation. The UFA 18:2 $\Delta 9,12$ shares the same chain length as one of the authentic products of the desaturase, having in common the $\Delta 9-10$ double bond as both products, and a similar melting temperature as palmitoleic acid. However, as $18: 2 \Delta 9,12$ is a polyunsaturated fatty acid, it is quite different structurally. The UFA $17: 1 \Delta 10$ does not have a $\Delta 9-10$, but its double bond is the same distance from the methyl end as 16:1 $\Delta 9$ 's bond. This nonnative UFA shares a similar melting temperature as 18:1 $\Delta 9$. In addition, we examined conditions that alter membrane fluidity to try and detect a consistent molecular response.

\section{Methods}

2.1. Strains, Media, and Growth Conditions. The yeast strain W303-1A (MATa, trp1-1, his3-11,15, ade2-1, leu2-3,112, ura3-1) was used in this study. Standard methods for yeast growth and media were as described [29]. YNBt medium is the standard synthetic complete medium $(0.67 \%$ yeast nitrogen base, $2 \%$ dextrose) with $0.1 \%$ or $1 \%$ tergitol NP-40 (Sigma) as noted, supplemented with appropriate amino acids, uracil and adenine. Fatty acids were prepared as stock solutions at 50 or $100 \mathrm{mM}$ concentration in ethanol and supplemented to YNBt media as noted. YNBt media without fatty acids contained 1\% ethanol.

Yeast transformations were performed via the lithium acetate procedure [30] using reagents from Takara Bio. For routine propagation of plasmids, E. coli NEB5 Alpha (New England Biolabs) were used, grown in LB medium supplemented with ampicillin. Standard procedures for cloning, growth and selection of bacteria were followed [31]. The plasmid p62 has placed lacZ under control of a 1015-bp HindIII/ SalI fragment of the OLE1 gene, starting from base -934 relative to the start codon, and including the first 27 codons of the desaturase placed in frame with lacZ [32].

2.2. Fatty Acid Analysis. To analyze total fatty acids, cells were cultured at $30^{\circ} \mathrm{C}$ in $200 \mathrm{ml} \mathrm{YNBt}$ overnight to a maximum density of $1-2 \times 10^{7}$ cells $/ \mathrm{ml}$. Fatty acids in ethanol (or ethanol alone for control) were added to cultures and incubation continued for four hours. Cells were harvested by centrifugation, washed with sterile water, and lipids were saponified and converted to methyl esters by a modified Bligh-Dyer method [33] Samples of fatty acid methyl esters were analyzed by gas chromatography on a Hewlett-Packard model 6890 gas chromatograph using a $30 \mathrm{~m} \mathrm{HP}-5$ column. Instrument conditions were as described [34]. Identification of cellular fatty acid species was made by comparison of retention times to those of authentic fatty acid standards (Sigma).

2.3. Beta-Galactosidase Assays. Yeast bearing plasmid p62 were grown overnight in YNBt media with either $1 \%$ or $0.1 \%$ tergitol as noted. Cultures were inoculated into fresh media with either $1 \%$ ethanol (for control) or noted UFA and incubated at $30^{\circ} \mathrm{C}$, shaking, for four hours before performing assays. Cells were harvested by centrifugation and $\beta$-galactosidase activity was measured using the permeablized cell method [29]. Enzyme activity was determined using Equation (1).

$\beta-$ galactosidase activity $=\frac{\mathrm{A} 420}{(\mathrm{~A} 600)(\text { volume of culture tested })(\text { time })}$.

Assays for each construct were performed on at least five replicates. Activity was normalized to the ethanol controls (set at $100 \%)$.

2.4. Fatty Acid Melting Point Determination. 99\% pure cis-10-heptadecenoic acid (Sigma) was loaded into three separate capillary tubes, melted, and centrifuged at about $2000 \mathrm{rpm}$ for two minutes. The samples were then allowed to solidify. Using a Barnstead International model 1001D MelTemp melting point apparatus, a preliminary "fast" reading was taken (increase of $5-10^{\circ} \mathrm{C} / \mathrm{min}$ ) to determine the general range of the melting point of the sample. The instrument was then cooled to $10^{\circ} \mathrm{C}$ below estimated melting point, and a second sample was read at a slow rate (increase of $1^{\circ} \mathrm{C} /$ 
min). The slow rate read was repeated. As a control, the melting temperature range of $99 \%$ pure cis- 9 octadecenoic acid was determined through this same method and found to be $13.0-13.4^{\circ} \mathrm{C}$, which matched the published value of $13.4^{\circ} \mathrm{C}$ well [35].

\section{Results}

3.1. The Relative Intracellular Concentration of Exogenous UFAs Can Be Controlled by the Amount of Tergitol in the Medium. In many experiments involving feeding of fatty acids to yeast cells, a compound with detergent properties, such as Tween or tergitol, is added to the growth medium to help keep the fatty acids solubilized and available to the cells. We hypothesized that the effective concentration of supplied fatty acids available to the cells may be controlled by the amount of detergent in the medium. We tested the effect of the amount of tergitol in the growth medium (at $0.1 \%$ and $1 \%$ ) on the relative incorporation of a fed fatty acid, which was maintained at a concentration of $1 \mathrm{mM}$. As seen in Figures 1(a) and 1(b), the relative amount of fatty acid that was taken up was dependent on the particular species. But in each case, more of the fed fatty acid was found in the cell at $0.1 \%$ tergitol growth conditions than at $1 \%$ tergitol (Figure 1(c)).

By examining the fatty acid profiles, we determined that when cells are not supplemented with UFA, the desaturase is active, resulting in a large proportion of total fatty acids being unsaturated; $16: 1 \Delta 9$ representing approximately $45 \%$ of total fatty acids, and $18: 1 \Delta 9$ about $30 \%$. When supplied $16: 1 \Delta 9$ or 18:1 $\Delta 9$, the fed fatty acid increases at the expense of the other. For example, when fed 16:1 $\Delta 9$, we can clearly see that the amount of 18:1 $\Delta 9$ produced by the desaturase has decreased substantially (Figures 1(a) and 1(b)). When we compare the $1 \%$ and $0.1 \%$ tergitol conditions in the case of $16: 1 \Delta 9$ feeding, we observe $16: 1 \Delta 9$ is a robust $85 \%$ of total fatty acids in the $0.1 \%$ tergitol condition, while approximately $55 \%$ of total fatty acids is $16: 1 \Delta 9$ in the $1 \%$ tergitol culture conditions. Both increases are basically at the expense of $18: 1 \Delta 9$, which decreased from the unfed condition of about $30 \%$ of total fatty acids to about $19 \%$ in the $1 \%$ tergitol condition and to $2 \%$ in the $0.1 \%$ tergitol condition. As it is impossible to distinguish under these conditions between native, endogenously produced $16: 1 \Delta 9$ and fed $16: 1 \Delta 9$, it is formally possible that the desaturase is still actively producing $16: 1 \Delta 9$ when fed $16: 1 \Delta 9$. However, the loss of production of $18: 1 \Delta 9$ indicates that most likely the desaturase itself is not active. This inability to distinguish endogenous from exogenous is not a problem in the feeding of the nonnative fatty acids $17: 1 \Delta 10$ and 18:2 $\Delta 9,12$. When these fatty acids are fed, we see concomitant decreases in both $16: 1 \Delta 9$ and $18: 1 \Delta 9$, indicating clearly that the desaturase is not as active under these fed conditions. Interestingly, we observed that 18:2 $\Delta 9,12$ is incorporated at a higher level than $17: 1 \Delta 10$ under both culture conditions; about $21 \%$ $18: 2 \Delta 9,12$ vs. $9 \% 17: 1 \Delta 10$ in the $1 \%$ tergitol conditions, and $69 \% 18: 2 \Delta 9$ vs. $35 \% 17: 1 \Delta 10$ in the $0.1 \%$ tergitol.

\subsection{Regulation of Expression from the OLE1 Promoter Is} Dependent on Fatty Acid Species and Concentration. In order to quantify the effect of growth in UFA supplemented medium on OLE1 transcriptional regulation, we examined the effects of the supplement on a reporter construct of the OLE1 promoter controlling expression of lacZ. Growth in medium containing $16: 1 \Delta 9$ or $18: 2 \Delta 9,12$ resulted in a steep decline in activity of $\beta$-galactosidase as compared to unsupplemented condition, while there was an intermediate level of activity in 18:1 $\Delta 9$ and 17:1 $\Delta 10$ supplemented conditions (Figure 2 ).

When we examined the influence of a higher effective concentration of UFA availability in the $0.1 \%$ tergitol condition versus $1 \%$ tergitol, in general, we observed that increasing the concentration of UFA increases its effect on the OLE1 promoter activity. However, 18:1 $\Delta 9$ does not follow this trend, exhibiting similar decreases in expression despite differences in intracellular amounts.

\subsection{Conditions That Alter Membrane Fluidity Have Unexpected}

Effects on OLE1 Expression. Examining the evidence so far, we hypothesized that the sensor(s) that regulate $O L E 1$ are responding to membrane fluidity signals. This is consistent with the idea that when fed a UFA with a lower melting temperature, it would result in increased membrane fluidity, creating a need to decrease expression of OLE1. To test this hypothesis, we examined the effect of other conditions that would affect fluidity on OLE1 expression; growth temperature and the presence of saturated or trans fatty acids.

Using the OLE1-lacZ reporter construct, wild type cells were grown in noted condition for four hours, after which $\beta$-galactosidase activity was measured. As seen in Figure 3(a), the effect of growth in saturated or trans $\Delta 9$ UFAs was examined for effect on OLE1 expression, and in Figure 3(b), the effect of temperature was examined on OLE1 expression. One would predict that in rigidifying conditions, expression of the desaturase would be induced and in fluidizing conditions, expression would be repressed. However, rigidifying conditions of growth in saturated fatty acids resulted in no significant change in reporter activity, while growth in a trans UFA significantly decreased OLE1 expression about $50 \%$ (Figure $3(\mathrm{a}))$. Indeed, growth at colder temperature $\left(24^{\circ} \mathrm{C}\right)$ actually resulted in about $50 \%$ decrease in OLE1 transcriptional activity as compared to the standard $30^{\circ} \mathrm{C}$ growth temperature condition (Figure 3(b)) while there was no significant difference in reporter activity between standard growth temperature of $30^{\circ} \mathrm{C}$ and $34^{\circ} \mathrm{C}$ (Tukey's range test, $p<.05$ ).

Table 1 provides physical/structural properties of the fatty acids tested and summarizes the effects of the different fatty acids for their ability to regulate $O L E 1$ transcriptional activity as measured in the reporter assay.

\section{Discussion}

The uptake and use of fatty acids from the diet into the cell is a critical step, necessary for energy use and storage, and as precursors for complex lipid formation. However, as there is wide diversity in fatty acid structure, this may result in some fatty acids being ideal and some being less optimal for use under particular growth or environmental conditions. While other studies have investigated the role of exogenous fatty acids in 


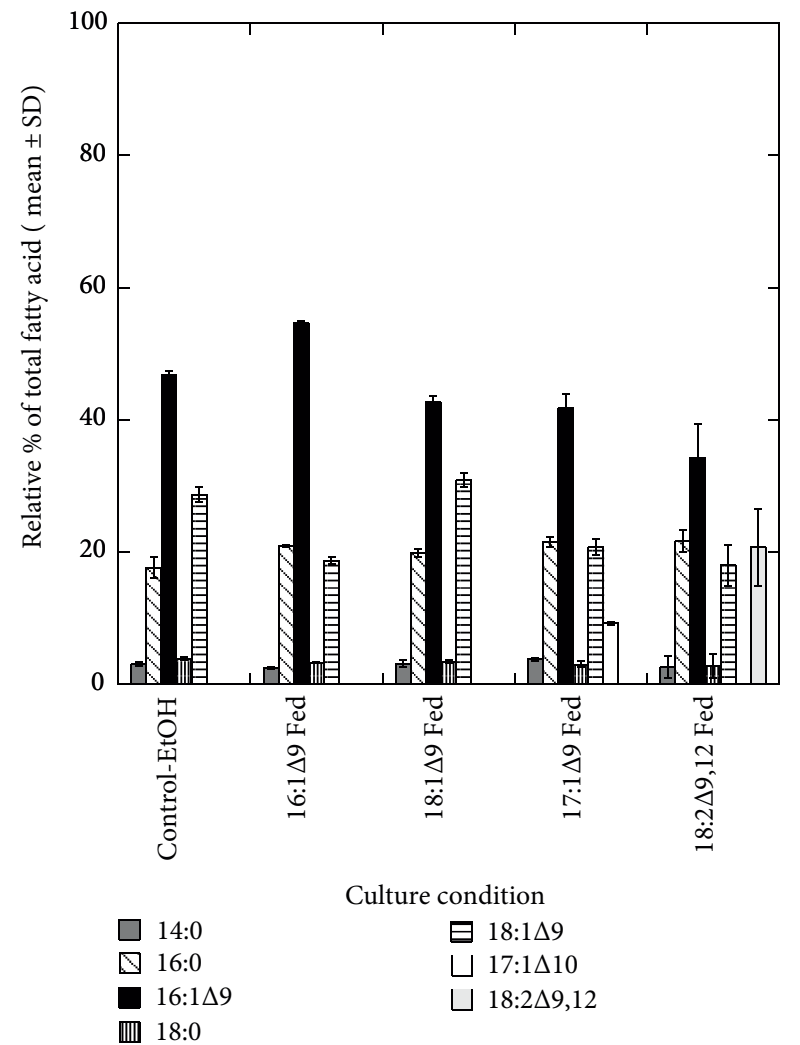

(a)

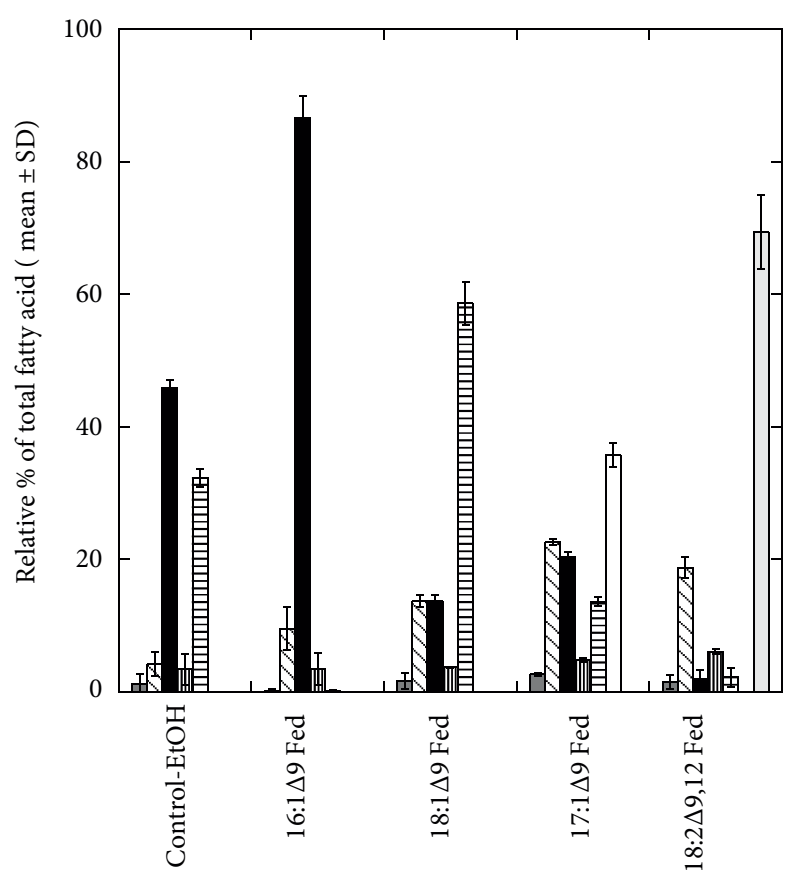

Culture condition

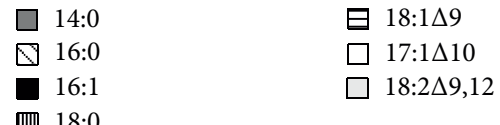

(b)

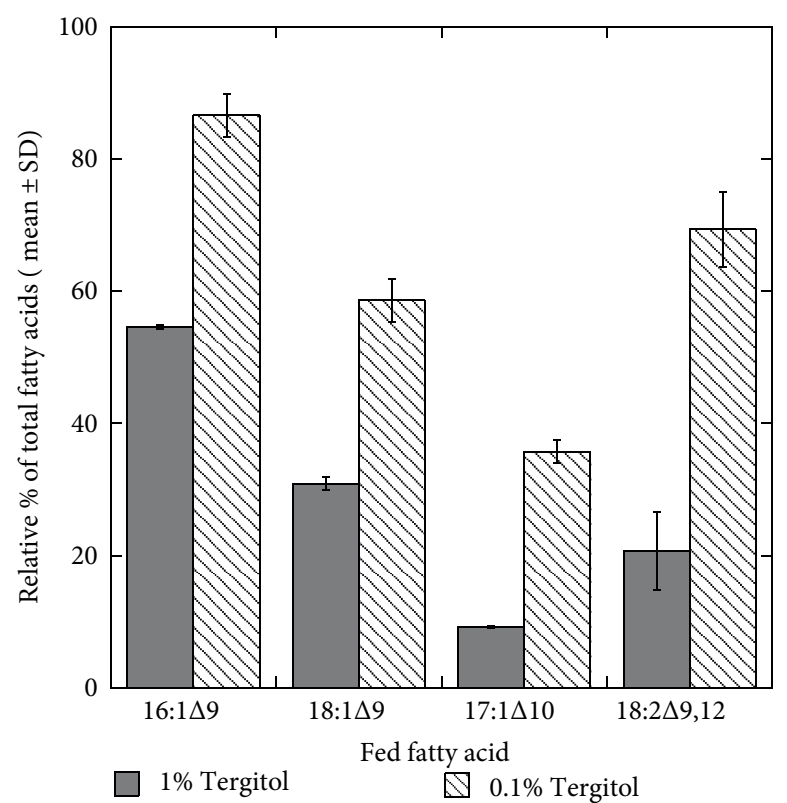

(c)

FIgURE 1: Fatty acid composition of cells grown \pm unsaturated fatty acids. Strain W303-1A was grown overnight and refreshed into YNBt medium with either $1 \%$ tergitol or $0.1 \%$ tergitol, containing $1 \mathrm{mM}$ noted fatty acid, or ethanol for control, for four hours. Cells were harvested, fatty acid methyl esters prepared and analyzed by GC as described in Section 2. At least three replicates were analyzed for each growth condition. Fatty acid profiles of cells grown in the presence or absence of unsaturated fatty acid supplements in either (a) $1 \%$ tergitol or (b) $0.1 \%$ tergitol. (c) A comparison of the relative amount of fed UFA present in $1 \%$ and $0.1 \%$ tergitol culture conditions.

regulation of OLE1, we also varied the effective amount of UFA available to cells by varying the amount of tergitol. This allowed us to examine both the effect of intracellular concentration and the structural differences on regulation. We found that cells fed $16: 1 \Delta 9$ or $18: 2 \Delta 9,12$ resulted in strongest regulation of desaturase expression. While it may not be surprising that supplying 


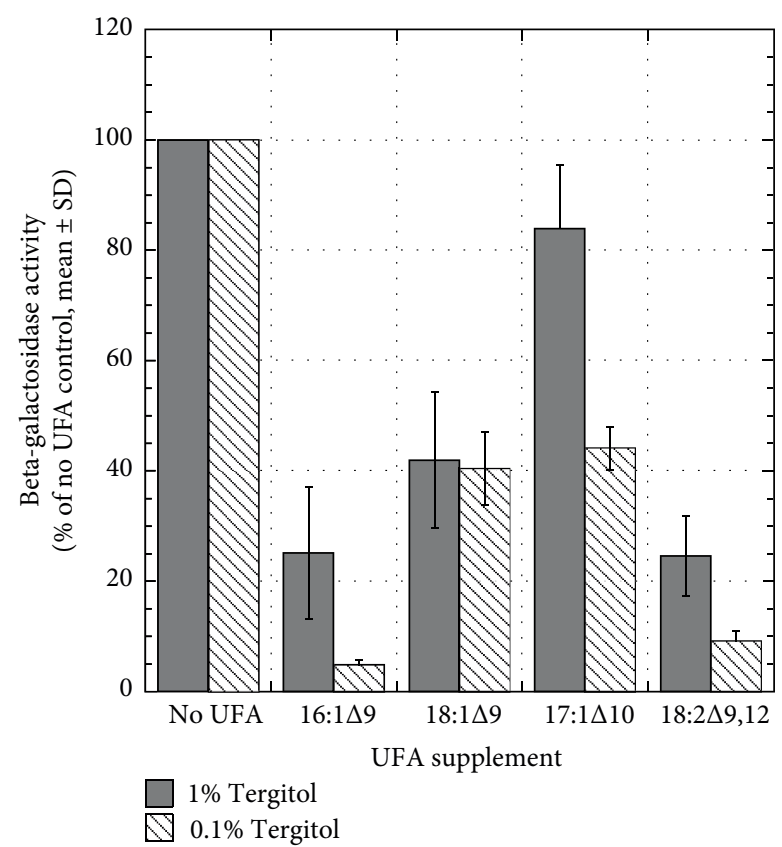

FIGURE 2: Effect of UFA species and tergitol concentration growth conditions on OLE1 expression. Wild type strain W303-1A with plasmid p62 (OLE1 promoter-lacZ reporter) was grown overnight, and refreshed into YNBt medium with either $1 \%$ tergitol or $0.1 \%$ tergitol, containing $1 \mathrm{mM}$ noted fatty acid, or $1 \%$ ethanol for control, for four hours. Cells were harvested and $\beta$-galactosidase activity determined. Activity was normalized to ethanol control. At least 5 replicates were performed for each growth condition.

a product of the desaturase, $16: 1 \Delta 9$, or even that a fatty acid that is somewhat similar to the monounsaturated product $18: 1 \Delta 9$, such as 18:2 $\Delta 9,12$ resulted in down regulation of the desaturase, it was unexpected that when fed 18:1 $\Delta 9$, an authentic product of the desaturase, we observed an intermediate level of regulation. The presence of 17:1 $\Delta 10$ led to a similar, tempered response as $18: 1 \Delta 9$. Taken together, these results leave us to question "what is the actual signal for down regulation of expression of OLE1?".

The regulation of OLE1 is not simply by the products of the desaturase as evidenced by the strong repression by $18: 2 \Delta 9,12$, or simply the presence of a $\Delta 9$ double bond, as $18: 1 \Delta 9$ would then cause the same degree of effect as $16: 1 \Delta 9$ or $18: 2 \Delta 9,12$. When we examine the physical properties of these tested fatty acids (Table 1), they differ in mass, double bond number and location, but none of those properties seem to be correlated to the differences in regulation. We ruled out the possibility that that the sensor is measuring from the methyl end, as the double bond in both 17:1 $\Delta 10$ and $16: 1 \Delta 9$ are the same distance ( 7 carbons) from the methyl end. However, there is a correlation between melting temperatures and the degree of regulation (Table 1).

Excellent work by Covino et al. have uncovered that part of the signaling occurs through Mga2p's ability to sense the lipid environment through formation of different transmembrane helical domain interactions [28]. These different domain interactions then affect the susceptibility of Mga2p to be processed into the p90 active form. Given that finding and our results

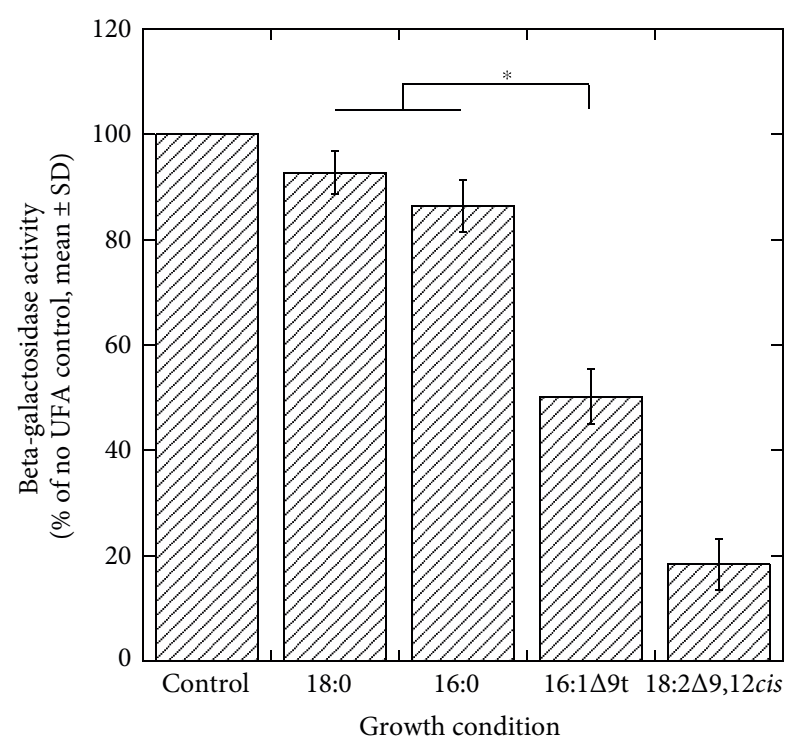

(a)

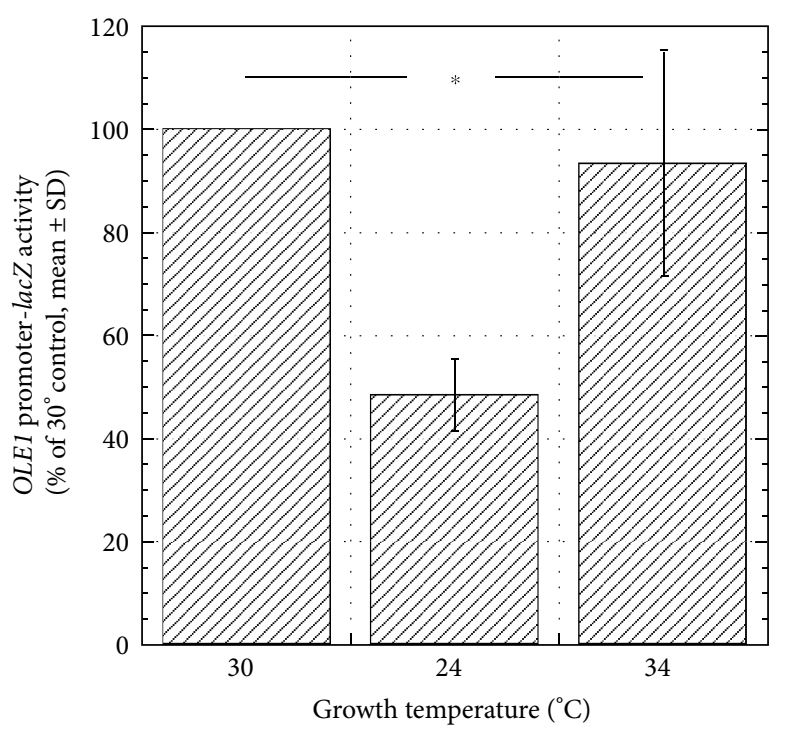

(b)

Figure 3: Effect of membrane altering conditions on OLE1 expression in wild type cells. Conditions thought to affect membrane fluidity were examined. Wild type W303-1A cells containing the OLE1-lacZ $p 62$ reporter plasmid were grown overnight in YNBt ( $1 \%$ tergitol) and refreshed into noted condition for four hours. Cells were harvested and $\beta$-galactosidase activity determined. At least 5 replicates were performed for each growth condition. (a) Effect of growth on OLE1 expression in minus ("control") or plus saturated or trans fatty acids (18:2 $\Delta 9,12$ cis is included as positive control). (b) Effect of temperature was examined on $O L E 1$ expression. $30^{\circ} \mathrm{C}$ is the normal growth temperature of yeast under laboratory conditions, and was used as the "control" condition. Asterisk indicates significant difference $(p<0.05)$.

described so far, we then hypothesized that the regulatory system responsible for determining the need for expression of the desaturase is actually monitoring the membrane environment condition as opposed to presence or absence of particular molecules; in other words, the physical status of the membrane may be important to the transcriptional regulation of the OLE1 gene. 
TABLE 1: Correlation of physical and structural properties of the fatty acids used in this study with regulatory effect on OLE1. For most fatty acids, the melting points were as reported [35]. The melting point of cis-10-heptadecenoic acid was determined as described in Section 2.

\begin{tabular}{|c|c|c|c|c|c|c|}
\hline Compound & $\begin{array}{l}\text { Number of } \\
\text { carbons }\end{array}$ & MW (daltons) & $\begin{array}{l}\text { Number/type of } \\
\text { double bonds in } \\
\text { acyl chain }\end{array}$ & $\begin{array}{c}\text { Position of } \\
\text { bond(s) from } \\
\text { carboxy end }\end{array}$ & $\begin{array}{l}\text { Melting point } \\
\left({ }^{\circ} \mathrm{C}\right)\end{array}$ & $\begin{array}{l}\text { Ability to regulate } \\
\text { OLE1 expression } \\
\text { (reporter gene } \\
\text { assay) }\end{array}$ \\
\hline $\begin{array}{l}\text { cis-9 hexadecenoic } \\
\text { acid }(16: 1 \Delta 9)\end{array}$ & 16 & 254 & $1 / c i s$ & $9-10$ & $-0.1^{\circ}$ & +++ \\
\hline $\begin{array}{l}\text { cis-9 octadecenoic } \\
\text { acid }(18: 1 \Delta 9)\end{array}$ & 18 & 282 & $1 /$ cis & $9-10$ & $13.4^{\circ}$ & ++ \\
\hline $\begin{array}{l}\text { cis,cis-9,12 } \\
\text { octadecadienoic } \\
\text { acid }(18: 2 \Delta 9,12) \\
\end{array}$ & 18 & 280 & $2 / c i s$ & $9-10,12-13$ & $-12^{\circ}$ & +++ \\
\hline $\begin{array}{l}\text { cis-10 heptadece- } \\
\text { noic acid }(17: 1 \Delta 10)\end{array}$ & 17 & 268 & $1 / c i s$ & $10-11$ & $15.7-15.9^{\circ}$ & ++ \\
\hline $\begin{array}{l}\text { Hexadecanoic acid } \\
(16: 0)\end{array}$ & 16 & 256 & 0 & NA & $145^{\circ}$ & 0 \\
\hline $\begin{array}{l}\text { trans-9 hexadece- } \\
\text { noic acid }(16: 1 \Delta 9 \\
\text { trans) } \\
\end{array}$ & 16 & 254 & $1 /$ trans & $9-10$ & $33-34^{\circ}$ & + \\
\hline $\begin{array}{l}\text { Octadecanoic acid } \\
(18: 0)\end{array}$ & 18 & 284 & 0 & $\mathrm{NA}$ & $67^{\circ}$ & 0 \\
\hline
\end{tabular}

However, when we examined the effect of conditions that we predict would have a rigidifying effect, lower growth temperature, or growth with saturated or trans fatty acids, we did not see the expected increase in $O L E 1$ gene expression. Additionally, growth at elevated temperatures, a condition that should increase membrane fluidity, did not decrease OLE1 gene expression. While these culturing conditions may have had other effects on the cell that could impact OLE1 expression, such as changing growth rate, they are consistent in their lack of support of a membrane fluidity sensor solely controlling OLE1. While it seems that membrane status is crucial, and is dependent on many considerations, including UFA content, our data indicate that it must not be simply the fluidity of the membrane, as conditions that affected fluidity did not fully impact regulation. These results imply that at least one part of the sensing system is not solely examining the physical status of the membrane and may be more specifically responding to a feature of the unsaturated fatty acid molecule. Further work is required to identify the nature of the sensing system.

\section{Data Availability}

The chromatographic data to support the findings of this study are available from the corresponding author upon request.

\section{Conflicts of Interest}

The authors declare that they have no conflicts of interest.

\section{Acknowledgments}

We thank Dr. J. Stukey for helpful discussions, and Dr. Jason Gilmore of the Hope College Department of Chemistry for guidance and use of the GC. Research reported in this publication was supported by the National Institute of General Medical Sciences of the National Institutes of Health under Award Number R15GM132853. The content is solely the responsibility of the authors and does not necessarily represent the official views of the National Institutes of Health. This work also was supported by the National Science Foundation [grant number REU DBI-0754293] and by a Hope College Jacob E. Nyenhuis Faculty Development Grant.

\section{References}

[1] J. E. Stukey, V. M. McDonough, and C. E. Martin, "Isolation and characterization of $O L E 1$, a gene affecting fatty acid desaturation from Saccharomyces cerevisiae," Journal of Biological Chemistry, vol. 264, no. 28, pp. 16537-16544, 1989.

[2] J. E. Stukey, V. M. McDonough, and C. E. Martin, "The OLE1 gene of Saccharomyces cerevisiae encodes the delta 9 fatty acid desaturase and can be functionally replaced by the rat stearoylCoA desaturase gene," Journal of Biological Chemistry, vol. 265, no. 33, pp. 20144-20149, 1990.

[3] Y. Lou and J. Shanklin, "Evidence that the yeast desaturase Ole1p exists as a dimer in vivo," Journal of Biological Chemistry, vol. 285, pp. 19384-19390, 2010.

[4] D. K. Bloomfield and K. Bloch, "The formation of $\Delta 9$-unsaturated acids," Journal of Biological Chemistry, vol. 235, no. 2, pp. 337-345, 1960.

[5] S. Irshad, A.-1. Mahul-Mellier, N. Kassouf, A. Lemarie, and S. Grimm, "Isolation of ORCTL3 in a novel genetic screen for tumor-specific apoptosis inducers," Cell Death \& Differentiation, vol. 16, no. 6, pp. 890-898, 2009.

[6] G. Abuali, W. Chaisaklert, E. Stelloo et al., "The anticancer gene ORCTL3 targets stearoyl-CoA desaturase-1 for tumourspecific apoptosis," Oncogene, vol. 34, no. 13, pp. 1718-1728, 2014. 
[7] C. A. Shively, M. J. Eckwahl, C. J. Dobry, D. Mellacheruvu, A. Nesvizhskii, and A. Kumar, "Genetic networks inducing invasive growth in Saccharomyces cerevisiae identified through systematic genome-wide overexpression," Genetics, vol. 193, no. 4, pp. 1297-1310, 2013.

[8] C. Gallo-Ebert, J. Francisco, H.-Y. Liu et al., "Mice lacking ARV1 have reduced signs of metabolic syndrome and nonalcoholic fatty liver disease," Journal of Biological Chemistry, vol. 293, no. 16, pp. 5956-5974, 2018.

[9] B. M. Vincent, D. F. Tardiff, J. S. Piotrowski et al., "Inhibiting stearoyl-CoA desaturase ameliorates a-synuclein cytotoxicity," Cell Reports, vol. 25, no. 10, pp. 2742-2754.e31, 2018.

[10] C. E. Martin, C.-S. Oh, and Y. Jiang, "Regulation of long chain unsaturated fatty acid synthesis in yeast," Biochimica et Biophysica Acta (BBA)-Molecular and Cell Biology of Lipids, vol. 1771, no. 3, pp. 271-285, 2007.

[11] S. Ballweg and R. Ernst, "Control of membrane fluidity: the OLE pathway in focus," Biological Chemistry, vol. 398, no. 2, pp. 215-228, 2016.

[12] M. A. Bossie and C. E. Martin, "Nutritional regulation of yeast delta-9 fatty acid desaturase activity," Journal of Bacteriology, vol. 171, no. 12, pp. 6409-6413, 1989.

[13] V. M. McDonough, J. E. Stukey, and C. E. Martin, "Specificity of unsaturated fatty acid-regulated expression of the Saccharomyces cerevisiae OLE1 gene," Journal of Biological Chemistry, p. 267, 1992.

[14] C. I. Gonzalez and C. E. Martin, "Fatty acid-responsive control of mRNA stability. Unsaturated fatty acid-induced degradation of the Saccharomyces OLE1 transcript," Journal of Biological Chemistry, vol. 271, no. 42, pp. 25801-25809, 1996.

[15] R. Chellappa, P. Kandasamy, C.-S. Oh, Y. Jiang, M. Vemula, and C. E. Martin, "The membrane proteins, Spt23p and Mga2p, play distinct roles in the activation of Saccharomyces cerevisiae OLE1 gene expression. fatty acid-mediated regulation of Mga2p activity is independent of its proteolytic processing into a soluble transcription act," Journal of Biological Chemistry, vol. 276, no. 47, pp. 43548-43556, 2001.

[16] K. M. You, C. Rosenfield, and D. C. Knipple, "Ethanol tolerance in the yeast Saccharomyces cerevisiae is dependent on cellular oleic acid content," Applied and Environmental Microbiology, vol. 69, no. 3, pp. 1499-1503, 2003.

[17] Y. Wang, S. Zhang, H. Liu, L. Zhang, C. Yi, and H. Li, “Changes and roles of membrane compositions in the adaptation of Saccharomyces cerevisiae to ethanol," Journal of Basic Microbiology, vol. 55, no. 12, pp. 1417-1426, 2015.

[18] Y. Jiang, M. J. Vasconcelles, S. Wretzel, A. Light, C. E. Martin, and M. A. Goldberg, "MGA2 is involved in the low-oxygen response element-dependent hypoxic induction of genes in Saccharomyces cerevisiae," Molecular and Cellular Biology, vol. 21, no. 18, pp. 6161-6169, 2001.

[19] H. Okuyama, M. Saito, C. J. Vasudev, S. Gunsberg, and S. Wakil, "Regulation by temperature of the chain length of fatty acids in yeast," Journal of Biological Chemistry, vol. 254, no. 24, pp. 12281-12284, 1979.

[20] Y. Nakagawa, N. Sakumoto, Y. Kaneko, and S. Harashima, "Mga2p is a putative sensor for low temperature and oxygen to induce OLE1 transcription in Saccharomyces cerevisiae," Biochemical and Biophysical Research Communications, vol. 291, no. 3, pp. 707-713, 2002.

[21] S. Zhang, Y. Skalsky, and D. J. Garfinkel, “MGA2 or SPT23 is required for transcription of the delta9 fatty acid desaturase gene OLE1, and nuclear membrane integrity in Saccharomyces cerevisiae," Genetics, vol. 151, no. 12, pp. 473-483, 1999.

[22] M. Rape, T. Hoppe, I. Gorr, M. Kalocay, H. Richly, and S. Jentsch, "Mobilization of processed, membrane-tethered SPT23 transcription factor by CDC48(UFD1/NPL4), a ubiquitinselective chaperone," Cell, vol. 107, no. 5, pp. 667-677, 2001.

[23] N. Shcherbik, T. Zoladek, J. T. Nickels, and D. S. Haines, “Rsp5p is required for ER bound Mga2p120 polyubiquitination and release of the processed/tethered transactivator Mga2p90," Current Biology, vol. 13, no. 14, pp. 1227-1233, 2003.

[24] N. Shcherbik and D. S. Haines, "Cdc48pNpl4p/Ufd1p binds and segregates membrane-anchored/tethered complexes via a polyubiquitin signal present on the anchors," Molecular Cell, vol. 25, no. 3, pp. 385-397, 2007.

[25] N. Kolawa, M. J. Sweredoski, R. L. J. Graham, R. Oania, S. Hess, and R. J. Deshaies, "Perturbations to the ubiquitin conjugate proteome in yeast ubx mutants identify Ubx2 as a regulator of membrane lipid composition," Molecular \& Cellular Proteomics, vol. 12, no. 10, pp. 2791-2803, 2013.

[26] M. A. Surma, C. Klose, D. Peng et al., "A lipid E-MAP identifies Ubx2 as a critical regulator of lipid saturation and lipid bilayer stress," Molecular Cell, vol. 51, no. 4, pp. 519-530, 2013.

[27] C. Rice, M. Cooke, N. Treloar, P. Vollbrecht, J. Stukey, and V. Mcdonough, "A role for MGA2, but not SPT23, in activation of transcription of ERG1 in Saccharomyces cerevisiae," Biochemical and Biophysical Research Communications, vol. 403, no. (3-4), pp. 293-297, 2010.

[28] R. Covino, S. Ballweg, C. Stordeur et al., "A eukaryotic sensor for membrane lipid saturation," Molecular Cell, vol. 63, no. 1, pp. 49-59, 2016.

[29] M. D. Rose, F. Winston, and P. Heiter, Methods in Yeast Genetics: A Laboratory Course Manual, Cold Spring Harbor Laboratory Press, Cold Spring Harbor, 1990.

[30] H. Ito, Y. Fukuda, K. Murata, and A. Kimura, "Transformation of intact yeast cells treated with alkali cations," Journal of Bacteriology, vol. 153, no. 1, pp. 163-168, 1983.

[31] J. Sambrook, E. F. Fritsch, and T. Maniatis, Molecular Cloning: A Laboratory Manual, Cold Spring Harbor Laboratory Press, NY, U.S.A, 2 edition, 1989.

[32] J. Y. Choi, J. Stukey, S. Y. Hwang, and C. E. Martin, "Regulatory elements that control transcription activation and unsaturated fatty acid-mediated repression of the Saccharomyces cerevisiaeOLE1 gene," Journal of Biological Chemistry, vol. 271, no. 7, pp. 3581-3589, 1996.

[33] E. G. Bligh and W. J. Dyer, "A rapid method of total lipid extraction and purification," Canadian Journal of Biochemistry and Physiology, vol. 37, pp. 911-917, 1959.

[34] V. McDonough, J. Stukey, and T. Cavanagh, "Mutations in erg4 affect the sensitivity of Saccharomyces cerevisiae to medium-chain fatty acids," Biochimica et Biophysica Acta (BBA)-Molecular and Cell Biology of Lipids, vol. 1581, no. 3, pp. 109-118, 2002.

[35] S. Budavari, Merck Index, Merck \& Co, 11th edition, 1989. 